\title{
Akzeleration der Prekarität: Analyse neuer Emanzipationsmodelle im kognitiven Kapitalismus mit Gilles Deleuze und Félix Guattari
}

\author{
Michael Feichtinger ${ }^{\star}$
}

\section{Zusammenfassung}

Ausgehend von Gilles Deleuzes und Félix Guattaris Weiterentwicklung der marxistischen Theorie des Kapitalismus in Tausend Plateaus lege ich dar, wieso bestimmte Widerstandsstrategien im Paradigma des kognitiven Kapitalismus nicht fruchtbar sein können und worin ihre Schwächen liegen. Im Anschluss an diese Kritik stelle ich Anna Tsings anthropologische Studie über die Matsutakepflücker_innen als ein Emanzipationsmodell vor, welches Transitionsmöglichkeiten in eine post-kapitalistische Gesellschaft in hyper-prekären Arbeitsbedingungen verortet. Die Arbeiter_innen von Amazons Mechanical Turk werden hierbei als äquivalentes Prekariat im kognitiven Kapitalismus diskutiert, da auch sie ihre eigene Destruktionslinie erschaffen. Abschließend zeige ich, dass der linke Akzelerationismus gewissermaßen eine in der Theorie selbst kaum explizierte Radikalisierung von Tsings Appell des Prekär-Werdens darstellt. Zusammenfassend soll damit eine mögliche Perspektive aufgezeigt werden, wie diese neue und spekulative Emanzipationsstrategie wieder an ein Ende des Kapitalismus und eine post-kapitalistische Welt denken lassen.

Schlagwörter: Deleuze, Guattari, Akzelerationismus, Prekarität, Kapitalismus

\section{Acceleration of Precarity: A Deleuzo-Guattarian Analysis of Novel Emancipation Strategies Within Cognitive Capitalism}

\section{Abstract}

Based on Gilles Deleuze's and Félix Guattari's deployment of Marx's theory of capitalism in Thousand Plateaus, I show why certain strategies of resistance within the new paradigm of cognitive capitalism cannot be successful and highlight their shortcomings. Following that critique, I introduce Anna Tsing's anthropological study on the matsutake pickers as a model of emancipation that maps possibilities for the transition to a post-capitalist society in hyper-precarious working conditions. I discuss the workers of Amazon's Mechanical Turk as an equivalent of the matsutake pickers within the paradigm of cognitive capitalism as they construct their line of destruction as well. Finally, I argue that the left accelerationism consists in a radicalization of Tsing's appeal of becomingprecarious that is hardly discussed in the theory itself. In the end I point out how this new and speculative emancipation strategies restore the possibility of thinking about the end of capitalism and a post-capitalist world.

Keywords: Deleuze, Guattari, Accelerationism, Precarity, Capitalism

\footnotetext{
* Michael Feichtinger, Universität Wien, Max F. Perutz Laboratories. E-Mail: michael.feichtinger@univie.ac.at Vielen Dank an Serjoscha Wiemer und Kurt Appel für die positive Resonanz zu einer früheren Version des Beitrags, sowie an die beiden anonymen Gutachter_innen von Momentum Quarterly für das konstruktive Feedback.
} 


\section{Das Ende des Kapitalismus?}

In seinem gleichnamigen Buch bezeichnet Mark Fisher den kapitalistischen Realismus als vorherrschenden Gemeinsinn unserer Zeit. Er charakterisiert dieses Phänomen treffenderweise als ,the widespread sense that not only is capitalism the only viable political and economic system, but also that it is now impossible even to imagine a coherent alternative to it" (Fisher 2009: 2). Die Ursachen dafür scheinen einerseits das Scheitern möglicher Gegenentwürfe zum Kapitalismus im 20. Jahrhundert sowie die Infiltration des Kapitalismus bis tief in unsere Freizeit zu sein. Der vermeintlich allumfassende Charakter des globalen Kapitalismus lässt klassische Emanzipations- und Widerstandsmodelle, die meist nur auf lokaler Ebene wirken und sich als Antagonist zum Kapitalismus positionieren, nicht mehr wirksam genug erscheinen und verlangt nach neuen Strategien, um mit der hegemonialen Vormachtstellung dieses Weltentwurfs umzugehen. Mein Fokus wird hier vor allem auf aktuellen Widerstandsmodellen in Bezug auf den sogenannten kognitiven Kapitalismus liegen, da aufgrund der Transformation durch dieses neue Paradigma viele neue emanzipative Ideen entstanden sind. Antonella Corsani spricht beispielsweise im Übergang vom Industriekapitalismus zum kognitiven Kapitalismus von Grundlegenden Transformationen in der Produktion, der Arbeitskraft, den Maschinen, dem Konsum und den Waren (Corsani 2012: 103-105).

Als Ausgangspunkt für meine Überlegungen werde ich zuerst zwei zentrale Punkte von Deleuze und Guattaris Weiterentwicklung der marxistischen Charakterisierung des Kapitalismus in Tausend Plateaus nachzeichnen. Um die Wirkmächtigkeit des Kapitalismus auf globaler Ebene zu verstehen, sollte dieser als globale Kriegsmaschine aufgefasst werden, welche durch ihre zugrunde liegende Axiomatik heterogene Realisierungsmodelle annehmen kann. Im ersten Teil wird es mir darum gehen, diese begriffliche Diagrammatik von Deleuze und Guattari zu konkretisieren und wichtige Konzepte für die weitere Analyse einzuführen. Dazu werde ich die Position von Michael Hardt und Antonio Negri, die in Empire eine Neubestimmung des Kapitalismus als kognitiven Kapitalismus mittels des theoretischen Rahmens von Deleuze und Guattari vornehmen, miteinbeziehen und parallel diskutieren. Außerdem nutze ich diesen Teil des Textes, um Divergenzen und Weiterentwicklungen im Vergleich zu Marx aufzuzeigen.

Innerhalb dieses theoretischen Rahmens werde ich im Weiteren argumentieren, wieso die vorgeschlage- nen Widerstandsstrategien im kognitiven Kapitalismus nicht fruchtbar sein können und worin ihre Schwächen liegen. Im Anschluss an diese Kritik stelle ich ausgehend von Anna Tsings anthropologischer Studie über die Matsutakepflücker_innen ein Emanzipationsmodell vor, welches Transitionsmöglichkeiten in eine post-kapitalistische Gesellschaft in äußerst prekären Arbeitsbedingungen verortet. Die Arbeiter_innen von Amazons Mechanical Turk werden hierbei als äquivalentes Prekariat im kognitiven Kapitalismus eingeführt, da auch sie ihre eigene Destruktionslinie erschaffen, sich also durch ihre Arbeit zur Schwelle der Kontroll- und Modulationsmechanismen hinbewegen. Im Anschluss werde ich den linken Akzelerationismus, wie er beispielsweise von Nick Srnicek und Alex Williams vertreten wird, nicht im Sinne einer technologischen Utopie, sondern als eine implizite Radikalisierung von Tsings Appell des Prekär-Werdens lesen. Unter Berücksichtigung von Judith Butlers Konzept der Prekarität werde ich abschließend diskutieren, worin das emanzipative Potenzial und die Gefahren der akzelerationistischen Hyper-Prekarisierung liegen. Zusammenfassend soll damit eine mögliche Perspektive aufgezeigt werden, wie diese neue Emanzipationsstrategie wieder an ein Ende des Kapitalismus und eine post-kapitalistische Welt denken lassen. Diese spekulative Perspektive zielt damit vor allem auf die Konstruktion einer offenen und gegenhegemonialen Zukunft durch die Beschleunigung von Destruktionslinien des globalen Kapitalismus. Damit stellt der hier vorgeschlagene Widerstand keine konkrete Utopie in Aussicht, sondern lässt die Kontingenz des Kapitalismus in Erscheinung treten und stört die hegemoniale Realität.

\section{Deleuzes und Guattaris globale Kriegsmaschine}

Deleuze und Guattari führen den Begriff der Kriegsmaschine in Tausend Plateaus im Zuge der Diskussion politischer Souveränität ein. Die Kriegsmaschine bildet hierbei sozusagen den De-jure-Gegenpol zum Staatsapparat, welchem sie immer entgegengesetzt ist und dessen Exteriorität sie verkörpert. Kriegsmaschine und Staatsapparat sind jedoch, wie sämtliche Begriffspaare dieser Art in den Tausend Plateaus, nicht als komplementäre Begriffe zu denken, sondern als zwei Vermögen bzw. Kräfte, die in einem bestimmten Gefüge unterschiedlich stark wirksam sind. Vereinfacht lässt sich sagen, dass die Kriegsmaschine das Außen des Staates darstellt. Wie sich im Weiteren zeigen wird, schließt dies jedoch nicht aus, dass sie auch aus dem 
Staat heraus wirksam werden und sich in diesen in veränderter Form integrieren kann. Verständlich wird die Flexibilität der weltweiten Kriegsmaschine vor allem durch ihr Potenzial, glatten Raum zu erschaffen.

\subsection{Der glatte und der gekerbte Raum}

Der glatte Raum zeichnet sich vor allem dadurch aus, dass er nicht als Ganzes konzeptualisiert und prädeterminiert werden kann, sondern nur Schritt für Schritt erkundbar ist, wobei er im Zuge dieser Erkundung erst geformt wird (Young et al. 2013: 289). Im Kontrast dazu steht der gekerbte Raum. Dieser kann als Ganzes bestimmt und konzeptualisiert werden. Es braucht dafür keine Erkundung des Raumes, da er als totale Entität durch seine internen Relationen bereits vorbestimmt ist. Dieses Phänomen des gekerbten Raumes findet sich im Schachspiel. Entscheidend dafür ist jedoch nicht die Einteilung des Spielfeldes in einzelne Quadrate, denn dies findet man auch beim Spiel Go, welches als Beispiel für den glatten Raum dienen kann. Das Spezifikum der Schachfiguren ist, dass sie codiert sind und damit „haben [sie] ein inneres Wesen oder ihnen innewohnende Eigenschaften“ (Deleuze/Guattari 1992: 483). Die Möglichkeiten bzw. die Identitäten der Dame oder des Turmes sind im Vorhinein festgelegt und während der Bewegung im „Spielraum“ nicht mehr veränderbar. Die Codierung einer jeden Schachfigur ergibt sich durch die inneren Relationen des Schachspiels, daher aus dem Verhältnis der einzelnen Figuren zueinander. Go-Steine besitzen im Vergleich dazu eine "anonyme Funktion“ (Deleuze/Guattari 1992: 483). Erst durch die Bewegung am Spielbrett wird dieses differenziert und die fluiden Identitäten der Spielfiguren bilden sich temporär heraus. Daraus resultiert, dass trotz der Tatsache, dass es nur vier einfache Grundregeln bei Go gibt, eine viel höhere Anzahl an Spielvarianten als beim Schach möglich ist. „Beim Schach wird der Raum codiert und decodiert, während Go ganz anders verfährt und den Raum territorialisiert und deterritorialisiert" (Deleuze/Guattari 1992: 483). Die Funktion der Steine im Go wird durch die spielerische Erschließung des Raumes permanent verändert. Man könnte auch von einer ständigen Verschiebung der Differenzen zwischen den Go-Steinen sprechen. Wichtig ist jedoch, dass zwischen glattem und gekerbtem Raum keine absolute Differenz besteht, da beides in seiner Reinform niemals existiert. Ein völlig glatter Raum würde die Auflösung in eine absolute Indifferenz meinen. Die begriffliche Unterscheidung zwischen den beiden ist jedoch dahingehend hilfreich, da sich damit Tendenzen angeben lassen, in die sich ein Gefüge entwickelt. Auf den Kapitalismus angewandt lässt sich diese Tendenz zur Präferenz eines glatten Raumes in der kontinuierlichen Erzeugung neuer Differenz für die Erschließung neuer Märkte wiederfinden. Ebenso gibt es keine festen Identitäten im Sinne eines gekerbten Raumes der Moderne, wie das Proletariat und die Kapitalisten, sondern eine fluide Verschiebung von Differenzen und Polarisierung zwischen den beiden Polen im neoliberalen Individuum.

Versteht man nun die Kriegsmaschine als ein Außen der staatlichen oder auch despotischen Ordnung, welche sich allem voran durch Codierung auszeichnet, so stellt sie im gekerbten Raum des Staatsapparates wieder glatten Raum her. In Empire bezeichnen Hardt und Negri mit dem titelgebenden Begriff die aktuellen Auswüchse des Kapitalismus im beginnenden 21. Jahrhundert als neue Souveränität, welche als ein allumspannendes und dezentralisiertes Netzwerk, bestehend aus supra- und transnationalen Unternehmen, operiert (Hardt/Negri 2003: 10). Die Funktionsweise dieses Empire, das Hardt und Negri klar vom Imperialismus unterscheiden, zeichnet sich dadurch aus, dass Firmen- und Konzernaktivitäten nicht mehr „ein abstraktes Kommando errichten oder schlichten Raub und ungleichen Tausch organisieren. Sie strukturieren und artikulieren stattdessen unmittelbar Territorien und Bevölkerung." (Hardt/ Negri 2003: 46) Diese Veränderung zeigt somit einen Paradigmenwechsel und damit auch die Notwendigkeit neuer Kritik- und Widerstandsformen auf.

Ähnlich operierend wie das Empire bei Hardt und Negri lässt sich nun auch der globale Kapitalismus als Kriegsmaschine verstehen. Die Kriegsmaschine stellt den glatten Raum her, welcher hierbei jedoch nur als kurzlebiger Übergangszustand existiert, der katalytisch genutzt werden kann, um eine neue Kerbung des Raumes durchzuführen. Es findet hier jedoch keine Transition zu einer glatten und indifferenten Welt statt, also kein Umschlagen von einer gekerbten in eine glatte Welt, wie dies bei Hardt und Negri an manchen Stellen im Empire scheint. Es wäre hier korrekter davon zu sprechen, dass wir in einer postmodernen Kondition leben, in welcher sich Differenzen permanent verschieben und neu ausbilden. „Die Tendenz des Kapitals ist ein glatter Raum, den unkodierte Ströme, Flexibilität, beständige Modulation und tendenzieller Ausgleich definieren “ (Hardt/Negri 2003: 336; Hervorhebung M.F.). Wichtig ist im Zuge dessen der Fokus auf die Tendenz. Glatter Raum koexistiert immer schon mit dem Gekerbten. Die Divergenz zum grob gekerbten Raum der Moderne 
besteht in der neoliberalen Postmoderne jedoch darin, dass diese Kerbung bzw. Differenzierung sich weitaus feingliedriger und kurzlebiger ausgestaltet. Der starke Fokus auf das Individuum, der sich ebenso in der florierenden Identitätspolitik niederschlägt, sorgt für eine weitaus differenziertere und gleichzeitig flexiblere Kerbung. Die Operationsweise dieses neuen Paradigmas besteht in der Kontrolle von Differenzen (Hardt/Negri 2003: 348).

Der globale Kapitalismus als eine „weltweite Kriegsmaschine stellt einen glatten Raum wieder her, um die Erde zu umspannen und einzuschließen“ (Deleuze/ Guattari 1992: 585). Im Zuge dessen schafft diese Kriegsmaschine damit das Globale als universale Kategorie der Kontrolle. Während für Marx und Engels noch der Staat derjenige ist, der die kapitalistischen Interessen organisiert, ist der Kapitalismus als weltweite Kriegsmaschine einer, der aus einem Netzwerk aus supra- und transnationalen Aktanten besteht, die somit außerhalb oder oberhalb staatlicher Souveränität operieren. Dieses Verhältnis ist jedoch kein dialektisches Innen und Außen mit dem Motor der Negativität als Triebkraft der Geschichte, sondern eine permanente immanente Differenzverschiebung innerhalb eines weltumspannenden Kapitalismus, der kein Außen mehr besitzt. Geschichte lässt sich dabei am ehesten in der Vorstellung eines Phasenraumes repräsentieren, in dem bestimmte neue Zustände zur Kapitalakkumulation besetzt werden. Damit einher geht auch, dass der Kapitalismus nicht mehr, wie bei Marx, extensiv expandieren kann. Als ein globaler und allumfassender Kapitalismus muss er intensiv expandieren (Hardt/Negri 2003: 283). Dies funktioniert durch die Einführung neuer Differenzen und gekerbter Räume, die zusätzliche Akkumulationsmöglichkeiten eröffnen. Dieses Phänomen lässt sich im Spätkapitalismus dahingehend beobachten, dass man sich kaum noch etwas vorstellen kann, das nicht potenziell in die Prozesse der Kapitalakkumulation eingeschleust werden kann. Daraus resultiert, „dass alle Ereignisse und Verhältnisse der Lebenswelt mit einem Marktwert ausgestattet werden könnten“ (Vogl 2010: 110). Dies zeigt sich unter anderem in einer „Komplizenschaft zwischen dem Ökonomischen und dem Biologischen" (Mbembe 2017: 19), die zu einer „Verwandlung der Menschen in belebte Dinge, in digitale Daten und Codes“ (Mbembe 2017: 20) führt. Damit wird das Leben selbst, inklusive des genetischen Codes, zum Thema der Ökonomie. Weiters erfolgt die intensive Expansion des Kapitalismus nicht nur mit Blick auf die Gegenwart, sondern es werden potenzielle zukünftige intensive Differenzierungen entworfen. Ein alltägliches Beispiel hierfür ist, dass Amazon nicht nur Artikel vorschlägt, die zu den eigenen Interessen passen, sondern ebenfalls solche, die auf zukünftige Präferenzen antworten, ihnen bestenfalls zuvorkommen. Dies führt zur ständigen Neuorganisation von Befriedigungen und Bedürfnissen durch die intensive Expansion des Kapitalismus. Jedoch realisiert sich der Kapitalismus nicht überall in der gleichen Form, sondern in heterogenen Ausprägungen. Dies funktioniert vor allem dadurch, dass der Kapitalismus mit einer Axiomatik operiert, wie es Deleuze und Guattari im Plateau zu den Vereinnahmungsapparaten beschreiben.

\subsection{Die Axiomatik des Kapitalismus}

Im Unterschied zu Marx bestimmen Deleuze und Guattari nicht mehr Gesellschaften durch ihre Produktionsweisen, sondern „durch maschinelle Prozesse“ (Deleuze/ Guattari 1992: 603). Mit dem Begriff der maschinellen Prozesse bezeichnen Deleuze und Guattari die Vermögen eines Gefüges. Die Produktionsweisen sind Resultate von den maschinellen Prozessen eines Gefüges. Es sind diese Prozesse, die bestimmte gesellschaftliche Formationen, wie beispielsweise primitive oder despotische Gesellschaften, hervorbringen und kein evolutionärer Fortschritt von der primitiven Gesellschaft über die despotische Gesellschaft zum Kapitalismus. Es ist hier wieder das oben erwähnte Bild des Phasenraums, der eine bessere Repräsentation geschichtlicher Prozesse darstellt als der Motor der Negativität. Es handelt sich dabei nur um eine Veränderung in der Ausprägung der jeweiligen Vermögen, die bestimmte Produktionsweisen hervorbringen.

Die Entstehung des Kapitalismus wird in Tausend Plateaus vom archaischen Staat ausgehend konstruiert. In diesem ist der Kapitalismus deswegen noch nicht möglich, weil der archaische Staat alle Ströme übercodiert (Adkins 2015: 225). Nun führt diese Übercodierung im archaischen Staat jedoch dazu, dass „dabei auch eine große Anzahl von decodierten Strömen“ (Deleuze/ Guattari 1992: 622) freigesetzt werden und dieser Übercodierung entgehen. Übercodierung bezeichnet dabei für Deleuze und Guattari die Unterordnung aller bestehender Codes unter ein transzendentes Prinzip, wie beispielsweise Gott oder den König. Es bedarf der Herstellung einer „kollektive[n] Figur eines Außenseiters“ (Deleuze/Guattari 1992: 622) durch den übercodierenden Apparat, damit die Strömungen erzeugt werden, welche für die Entstehung des Kapitalismus notwendig waren. Die Entstehung des Kapitalismus im Okzident ergibt 
sich daraus, dass „metallverarbeitende Handwerker und Händler im Westen einen ganz anderen Status bekommen, da sie in ihrer Existenz nicht unmittelbar von einem Überschuß abhängig sind, der von einem lokalen Staatsapparat akkumuliert wurde“ (Deleuze/Guattari 1992: 624). Dadurch taucht ein „ganz anderer Pol des Staates auf" (Deleuze/Guattari 1992: 625), welchen Deleuze und Guattari durch „Mischformen von Privatem und Öffentlichem“ (Deleuze/Guattari 1992: 625) charakterisieren. Dadurch entsteht für den Staatsapparat die Aufgabe, „die Verbindungen der als solche decodierten Strömungen zu organisieren“ (Deleuze/Guattari 1992: 625). Für die Vereinigung der beiden Ströme Reichtum und Arbeit muss der Staatsapparat diese nun dahingehend organisieren, dass einerseits die „Strömung der Arbeit nicht mehr durch Sklaverei oder Leibeigenschaft" (Deleuze/ Guattari 1992: 627) bestimmt wird und andererseits „der Reichtum nicht mehr als Grundeigentum, Finanz- oder Warenreichtum“ (Deleuze/Guattari 1992: 627) festgelegt wird. Nun konstituiert „die plötzliche abstrakte Vereinigung [der beiden Ströme] den Kapitalismus, indem sie [dem] einen ein universales Subjekt und [dem] anderen ein beliebiges Objekt gibt“" (Deleuze/Guattari 1992: 627). Diese stark abstrakte Struktur, die dadurch entsteht, ist auch der Grund für die axiomatische Organisation des Kapitalismus.

Mit der Axiomatik, welche die Übercodierung im Kapitalismus ersetzt, meinen Deleuze und Guattari eine Verbindung von decodierten Strömen, welche unmittelbar nur „rein funktionale Elemente und Beziehungen [betrifft], deren Wesen nicht spezifiziert ist und die direkt in ganz unterschiedlichen Bereichen gleichzeitig realisiert wird“ (Deleuze/Guattari 1992: 630). Genau deswegen ist Axiomatik auch nicht als Metapher zu verstehen. „Die Axiome des Kapitalismus sind [hierbei] weder theoretische Sätze noch ideologische Formeln, sondern operative Aussagen, die die semiologische Form des Kapitals bilden und als Bestandteile in die Gefüge der Produktion, Zirkulation und Konsumtion eingehen.“ (Deleuze/Guattari 1992: 640) Im Gegensatz dazu sind Codes immer lokal und spezifisch (Adkins 2015: 226). Die Übercodierung ordnet noch alles einer hierarchisch organisierten, transzendenten Instanz unter (Adkins 2015: 227), wie beispielsweise dem König oder Gott, wohingegen die Axiomatik in verschiedenen „Realisierungsmodelle[n]“ (Deleuze/Guattari 1992: 630) immanent ist. Weiters können operative Aussagen innerhalb der Axiomatik einfach hinzugefügt und entfernt werden. Damit ist gemeint, dass beispielsweise die „Wirtschaftstheorie von Keynes und der New Deal“
(Deleuze/Guattari 1992: 640) neue Axiome hinzugefügt haben, während der Nationalsozialismus sich „durch die Beseitigung des Binnenmarktes und durch die Reduktion von Axiomen“ (Deleuze/Guattari 1992: 640) auszeichnet. Da es kein festes transzendentes Prinzip, wie noch in der despotischen Staatsform gibt, erlaubt die Axiomatik eine viel größere Anpassungsmöglichkeit. Dies ermöglicht auch eine Isomorphie zwischen den heterogenen Realisierungsmodellen der kapitalistischen Axiomatik. Die Isomorphie kommt davon, dass alle „Realisierung[en] des Kapitals in Abhängigkeit von einem einzigen und gleichen [...] Weltmarkt" (Deleuze/Guattari 1992: 643) entstehen. Die Heterogenität resultiert daraus, dass verschiedene Realisierungsmodelle des Kapitalismus durch die oben beschriebene Flexibilität der Axiomatik möglich sind und in verschiedenste Lebenswelten Einzug halten können. So ist der Kapitalismus im Neoliberalismus ebenso möglich wie im Nationalsozialismus.

\subsection{Widerstand bei Deleuze und Guattari}

Deleuze und Guattari schließen das 13. Plateau zu den Vereinnahmungsapparaten mit einem Satz, der ihre Vorstellung des Widerstandes gegen den Kapitalismus in dieser axiomatischen Form zusammenfasst: „Es gibt keinen Kampf, der nicht inmitten all dieser unentscheidbaren Sätze geführt wird und der nicht revolutionäre Konnexionen gegen die Konjugationen der Axiomatik setzt." (Deleuze/Guattari 1992: 655) Die unentscheidbaren Sätze, welche Deleuze und Guattari dabei anführen, sind eine der „Gegebenheiten“ der kapitalistischen Axiomatik. Deleuze und Guattari bezeichnen damit, dass es innerhalb der kapitalistischen Axiomatik immer eine Koexistenz von etwas gibt, das dieser Axiomatik ,unaufhörlich entgeht, indem es Fluchtlinien folgt" (Deleuze/ Guattari 1992: 655). Diese systemimmanente Tendenz zur Veränderung bzw. zur Revolution, welche aus den decodierten Strömen resultiert, die der Axiomatisierung entgehen (Adkins 2015: 230), ist somit für Deleuze und Guattari in der kapitalistischen Axiomatik immer schon vorhanden. Daher wird klar, warum der Kampf zur Abschaffung des Kapitalismus innerhalb dieser unentscheidbaren Sätze geführt wird. Ein solcher Kampf ist somit einer, der im Kapitalismus immanent wirksam wird und nicht ein Außen als Antagonisten zu konstruieren versucht.

Das „Prinzip der Konnexion“ (Deleuze/Guattari 1992: 16) findet sich bereits in der programmatischen Einleitung von Tausend Plateaus als eines der Merkmale des Rhizoms. Bereits hier wird ersichtlich, dass das Prinzip 
der Konnexion sich einer baumartigen, hierarchischen Strukturierung entgegensetzt, da durch das Prinzip der Konnexion „[j]eder Punkt eines Rhizoms [...] mit jedem anderen verbunden werden“ (Deleuze/Guattari 1992: 16) kann und muss. Die revolutionäre Konnexion, von welcher also am Ende des 13. Plateaus die Rede ist, widersetzt sich auch der Eingliederung von Minderheiten in eine kapitalistische Axiomatik, weil ihnen deren Hierarchie nicht angemessen ist (Deleuze/Guattari 1992: 654). Viel eher geht es hierbei schon um eine Auflösung der Hierarchie und einer Konnexion der Fluchtlinien und deterritorialisierten Ströme im Sinne einer angestrebten rhizomatischen Organisationsweise. Hier lässt sich jedoch an Deleuze und Guattari die Anfrage stellen, ob der Kapitalismus in der Form, wie in Hardt und Negri beschreiben, nicht selbst schon eine rhizomatische Organisationsweise besitzt, die kein Zentrum mehr hat und als Netzwerk operiert. Jedoch lässt sich ein revolutionäres Rhizom vom kapitalistischen Rhizom dahingehend differenzieren, dass ersteres seine eigene Kontingenz affirmiert, wohingegen letzteres durch den Prozess der Konjugation eine scheinbare Stabilität erzeugt, die Fisher, wie eingangs erwähnt, als kapitalistischen Realismus bezeichnet. Der globale Kapitalismus durchläuft in dieser Form zwar eine permanente intensive Differenzierung, aber hält eine grundlegende Axiomatik stabil. Das revolutionäre Rhizom wäre deswegen eines, das sich keine neue Axiomatik gibt.

Im 9. Plateau führen Deleuze und Guattari die Unterscheidung von Konnexion und Konjugation dahingehend ein, dass erstere als Verbindung „die Art und Weise bezeichnet, in der decodierte und deterritorialisierte Strömungen sich wechselseitig Auftrieb geben“ (Deleuze/Guattari 1992: 301). Mit diesen decodierten und deterritorialisierten Strömungen, welche sich dabei Auftrieb geben, sind in dem genannten Fall nun die Fluchtlinien gemeint, welche sich aus der inhärenten Tendenz zur Veränderung innerhalb der kapitalistischen Axiomatik formen. Die Konjugation hingegen, welche den modus operandi für die Beständigkeit des Kapitalismus (Adkins 2015: 230) darstellt, vereinigt diese revolutionären Ströme, zeigt „ihren relativen Stillstand an, also eine Art von Kulminationspunkt, der nun die Fluchtlinien verstopft oder verschließt, eine allgemeine Reterritorialisierung bewirkt und die Strömungen der Vorherrschaft einer Strömung unterstellt, die sie übercodieren kann“ (Deleuze/Guattari 1992: 301). Die Konjugation erzeugt bzw. stabilisiert somit auch die Hegemonie der kapitalistischen Axiomatik. Dieser Prozess der scheinbaren Stabilität ist jedoch immer nur ein metastabiler und kontingenter, denn der „Weltmarkt braucht [...] den glatten Raum unkodierter und deterritorialisierter Ströme“ (Hardt/Negri 2003: 341), um, wie oben erwähnt, intensiv zu expandieren. Die Stabilität des Kapitalismus wird jedoch als absolut erlebt, womit sein Ende unvorstellbarer bleibt als das Ende der Welt (z.B. Fisher 2009: 1).

Der politische Widerstand scheint damit für Deleuze und Guattari immer schon im Keim der Axiomatik des Kapitalismus angelegt bzw. findet sich innerhalb dieser immer ein Einstiegspunkt, an welchem dieser Widerstand ansetzen kann. Hierzu schlagen Deleuze und Guattari vor, die vorhandenen schöpferischen Fluchtlinien auszunutzen, um in ein neues Gefüge überzutreten. Dadurch existiert auch nicht mehr ein klar definiertes Emanzipationssubjekt, das in einer dialektischen Beziehung zu seinem Antagonisten steht, wie dies bei Marx der Fall ist. Hardt und Negri aktualisieren Marx dahingehend, dass sie die Multitude als neues Subjekt der Emanzipation ausmachen. Die Multitude bezeichnet das subversive Vermögen gemeinsam handelnder Singularitäten, die für Hardt und Negri zwar bereits vorhanden sind, aber nur virtuell. Das Virtuelle bezieht sich hierbei mit Rückgriff auf Deleuze auf dasjenige, was zwar real, aber nicht aktual ist. Die Multitude ist für Hardt und Negri ein Produkt der Struktur des Empire und damit wie bei Deleuze und Guattari dem Kapitalismus immanent. Die Multitude ist schlussendlich der Begriff für „ein neues Proletariat“ (Hardt/Negri 2003: 409), womit all jene bezeichnet werden, „deren Arbeit vom Kapital ausgebeutet wird“ (Hardt/Negri 2003: 409). Dass ein derartiges Widerstandssubjekt unter anderen sich einer ähnlichen Problematik gegenüber sieht wie die Widerstandsansätze, die ich im kommenden Kapitel diskutieren werde, die versuchen, gerade diese sich bietenden decodierten Ströme aufzugreifen und produktiv zu nutzen, werde ich weiter unten noch ausführen. Was diese Widerstandsansätze vereint, ist, dass sie der erneuten Vereinnahmung durch die kapitalistische Kriegsmaschine nicht entgehen.

\section{Schöpferische Fluchtlinien und ihre Vereinnahmung}

Wie bereits eingangs erwähnt, liegt mein Fokus in diesem Abschnitt auf Fluchtlinien, die Theoretiker_innen im Aufkommen des neuen Paradigmas des kognitiven Kapitalismus verorten. Corsani sieht hierbei Transformationen von zentralen Elementen des Kapitalismus, woraus sich neue Emanzipationspotenziale entwickeln. 
3.1 Eine tiefgreifende Mutation des Kapitalismus?

Nach Corsani wird die Produktion von Waren im neuen Paradigma durch die Innovation als Produktion von Wissen ersetzt. Der zentrale Unterschied zur Innovation im Paradigma des Industriekapitalismus ist hier, dass die Produktion von Wissen, ergo die Innovation, nicht mehr hinsichtlich ihres Nutzens oder im Kontext der Warenproduktion verstanden werden kann, sondern als Produktion von Wissen für Wissen und nicht Wissen für bessere Warenherstellung. Letzteres wäre eine Gleichsetzung von Innovation mit technologischer Innovation, welche für Corsani im kognitiven Kapitalismus nicht mehr gültig ist. Weiters ist die Arbeitskraft im kognitiven Kapitalismus nicht mehr als eine spezifische Ressource zur Warenproduktion anzusehen, sondern verlangt, so Corsani, nach einem neuen Begriff von Arbeit, der „der Spezifität des Subjekts der kreativen Tätigkeit gerecht wird“ (Corsani 2012: 113). Dieses Subjekt ist nicht mehr ein Subjekt der Wiederholung, sondern des Schaffens. Diese Transformation ist eng verknüpft mit dem, was Deleuze treffend als Maschinen der dritten Art oder Informationsmaschinen bezeichnet, die den einfachen und energetischen Maschinen folgen (Deleuze 1993: 259). Während im Industriekapitalismus die Maschine hoch spezialisiert und heterogen war und die Arbeit homogen, hat sich dies im kognitiven Kapitalismus dahingehend gewandelt, dass durch die Informations- und Kommunikationstechnologien eine homogene Meta-Maschine entstanden ist, die heterogene, schaffende Arbeitssubjekte benötigt. Während also in der klassischen Fabrik des Industriezeitalters eine hoch spezialisierte mechanische Maschine vorhanden war, die relativ homogene Arbeitssubjekte erforderte, vollzieht sich im kognitiven Kapitalismus der Wandel hin zum Computer als MetaMaschine, deren Spezialisierung und Performance von den schaffenden Nutzer_innen abhängt. „Die Performance des technischen Geräts hängt von der Intelligenz, der Kreativität und Erfindungskraft der lebendigen Arbeit ab, die als unmittelbar kooperativ erscheint" (Corsani 2012: 116). Damit verschiebt sich der determinierende Pol für das Endprodukt von der Maschine hin zum individuellen Menschen. Schlussendlich ist der Konsum von Wissen dahingehend grundlegend verschieden von dem der klassischen Ware, dass es sich nicht verbraucht. Weiters ist der Konsum oft gleichzeitig wieder Produktion von neuem Wissen. Damit verlagert sich auch der Produktionsort des Wissens aus der Fabrik heraus. Diese Kombination birgt für Corsani ein derartiges post-kapitalistisches Potenzial, dass sich diese Veränderungen durch das neue Paradigma nicht mehr mit dem Kapitalismus zusammen denken lassen. Dies drückt sich bereits im Titel ihres Essays „Gibt es einen kognitiven Kapitalismus?" aus, welcher nicht nach der Existenz des neuen Paradigmas fragt, sondern danach, ob nach dem Bruch mit dem alten Paradigma überhaupt noch von Kapitalismus als solchem die Rede sein kann.

Die vergangenen Jahrzehnte haben jedoch gezeigt, dass es nicht zu einem Umsturz des Kapitalismus gekommen ist, sondern sich dieses sogenannte neue Paradigma durch „[p]rekäre Beschäftigung, Hyperausbeutung, hohe Mobilität und hierarchische Abhängigkeit" (Lazzarato 1998: 47) auszeichnet. Anstatt in ein neues Gefüge überzugehen, wurden die Axiomatik des Kapitalismus angepasst und die Fluchtlinien vereinnahmt. Es kam zum von Hardt und Negri beschriebenen Paradigmenwechsel innerhalb der kapitalistischen Axiomatik. Trotz grundlegender Transformationen scheint der Kapitalismus hier immer nur an die Grenze, nie aber an seine Schwelle zu kommen. Diese begriffliche Unterscheidung von Deleuze und Guattari bezeichnet hierbei folgenden Unterschied, wonach „die Grenze das Vorletzte bezeichnet, das einen notwendigen Neubeginn anzeigt, und die Schwelle das Letzte, das eine unvermeidliche Änderung anzeigt" (Deleuze/Guattari 1992: 607). Die Grenze stellt dasjenige dar, was ein Gefüge vertragen kann, ohne die Schwelle zu erreichen, die es „veranlassen würde, das Gefüge zu wechseln“ (Deleuze/Guattari 1992: 607). Ein ähnliches Problem, nämlich nie über die Grenze hinauszukommen, zeigt sich auch bei neueren Widerstandsmodellen, die sich aus der Onlinekultur entwickelt haben.

\subsection{Commons-basierte Peer-Produktion und Hackerkultur}

Yochai Benkler argumentiert in The Wealth of Networks dafür, dass es zwei große ökonomische Veränderungen in jüngster Vergangenheit gegeben hat:

„First, the basic output that has become dominant in the most advanced economies is human meaning and communication. Second, the basic physical capital necessary to express and communicate human meaning is the connected personal computer." (Benkler 2006: 32)

Es kam also, so Benkler, zu einer Veränderung in der Produktion hinsichtlich der oben bereits erwähnten Veränderung der Ware von physischen Erzeugnissen 
zu immateriellen Erzeugnissen. Außerdem führte die Wichtigkeit des Computers als homogene MetaMaschine $\mathrm{zu}$ einer Distribution von Produktion in Netzwerke und einer Dezentralisierung. Dadurch ergibt sich für Benkler die Möglichkeit einer neuen Modalität, um Produktion zu organisieren, nämlich jene der commons-basierten Peer-Produktion. Dies zeichnet sich durch folgende Charakteristika aus: „radically decentralized, collaborative, and nonproprietary; based on sharing resources and outputs among widely distributed, loosly connected individuals who cooperate with each other without relying on either market signals or managerial commands" (Benkler 2006: 60).

Christian Siefkes nimmt diese Idee von Benkler auf und formuliert aufbauend auf der Idee der Organisation der commons-basierten Peer-Produktion von Software, welche sich diesen Grundideen der Offenheit und Dezentralität verschrieben hat, die Möglichkeit einer konkreten Utopie und eines neuen Gesellschaftsentwurfs. Die vier leitenden Prinzipien für die post-kapitalistische Gesellschaft von Siefkes sind Kooperation auf Augenhöhe, Beitragen statt Tauschen, Commons und Besitz statt Eigentum sowie dezentralselbstheilend (Siefkes 2016: 64-66). Mit Kooperation auf Augenhöhe meint Siefkes hier nicht die flachen Hierarchien, die weite Verbreitung mit der Transition zum kognitiven Kapitalismus gefunden haben, sondern den freiwilligen Zusammenschluss von Kooperationspartner_innen. Dabei gibt es zwar aus Organisationszwecken „Koordinatorinnen [...], die ein Projekt auf Kurs halten und entscheiden, ob Beiträge integriert oder zurückgewiesen werden, aber diese sind auf die freiwilligen Beiträge der anderen angewiesen und können keinerlei Zwang anwenden“ (Siefkes 2016: 65). Mit dem Prinzip des Beitragens anstatt des Tauschens verweist Siefkes darauf, dass die Motivation für die Beteiligung an Projekten nicht nur vom Verkauf oder Tausch des Produktes angetrieben ist, wie dies in der Marktwirtschaft die Regel ist. Weiters entsteht durch die globale Vernetzung eine neue Art von Gemeingütern. Siefkes beschreibt dies wie folgt:

„Das Internet hat dieser uralten Praxis, die in Vergessenheit geraten, wenn auch nie ganz verschwunden war, zu einer Renaissance verholfen. Doch eins ist bei den digitalen Commons anders: Während bei traditionellen Allmenden die Gemeinschaft der Kümmerer und Nutzerinnen lokal begrenzt war - etwa auf die Bewohner eines bestimmten Dorfes -, sind diese neuen Allmenden grundsätzlich offen für alle, die mitmachen wollen und bereit sind, sich an kollektiv festgelegte Spielregeln zu halten."

(Siefkes 2016: 66)

Diese Annahmen mögen zwar für digitale Waren und Wissen zutreffend sein, ändern sich jedoch für Waren, die bei Gebrauch verbraucht werden oder die nur begrenzte Haltbarkeit haben. Siefkes postuliert zwar, dass sich dieses Prinzip auf alle Arten von Waren anwenden ließe, jedoch setzt er dazu einen gewissen Naturzustand des Menschen voraus, der nicht von nur im Kapitalismus geltenden Bedingungen „verdorben“ ist. Luxusgüter reduziert er hierbei beispielsweise rein auf ihren Prestigeeffekt (Siefkes 2016: 70).

Denkt man jedoch an die Transportunterschiede zwischen materiellen und immateriellen Waren sowie die bereits erwähnte begrenzte Haltbarkeit von einigen von diesen, so kommt es schnell wieder zur gewohnten Lokalität klassischer anarchistischer Ansätze, die die freie Verneinung von lokalen, selbstorganisierten Gruppen befürworten. Die Problematik besteht mit Hinblick auf die obige Analyse des Kapitalismus als weltweite Kriegsmaschine einerseits darin, dass angenommen wird, es gäbe ein Außen des Kapitalismus, in welchem sich ein derartiger lokaler Gesellschaftsentwurf etablieren könnte, ohne vereinnahmt zu werden. Andererseits sind die Ideen von Siefkes durch die letztendlich wieder durch lokale Begrenztheit und schwere Skalierbarkeit in Hinblick auf materielle Waren nicht in der Lage, sich gegen eine weltweite Hegemonie zu etablieren bzw. deren Allmacht und Stabilität in Frage zu stellen.

Der klare Fall der Vereinnahmung und Transformation derartiger Wissensproduktionsprozesse findet sich in der vielen Gratisarbeit, welche jeder User/ jede Userin von sozialen Netzwerken verrichtet. Auch hier wird in gemeinsamer Weise und ohne wirklichen Zwang Wissen produziert. Größtenteils wird dies gar nicht wirklich als Arbeit wahrgenommen, wie Andrew Ross anmerkt: „By far the majority of social network users are unaware of how the platform owners profit from the volunteer content of their communications, or indeed how they themselves are generating monetizable product for the owners" (Ross 2013: 18). In diesem Profitgewinn aus Wissensproduktion, an der sich viele Teilnehmer_innen aus Spaß, Neugierde oder auch Eigennutz beteiligen, lässt sich die Problematik von Siefkes Art des Widerstandes sehen. Das Problem sowohl von Benkler als auch bei Siefkes scheint darin zu liegen, dass beide Dezentralisierung als beinahe inhärent antikapitalistisch ansehen. Versteht man Kapitalismus jedoch in der oben angedeuteten Perspektive 
von Deleuze und Guattari bzw. Hardt und Negri, so operiert er durch den Paradigmenwechsel nicht mehr zentralisiert, sondern als ein dezentralisiertes Netzwerk. Durch die Flexibilität und Anpassungsfähigkeit einer Kriegsmaschine, die mit einer Axiomatik operiert, wird die radikale Distribution und Kooperation innerhalb commons-basierter Peer-Produktion zum Instrument der Kontrolle und Modulation von Subjekten vereinnahmt.

Diese Vereinnahmung von Ko-Kreation zur Modulation und Kontrolle von Konsumer_innen lässt sich nach Detlev Zwick, Samuel Bonsu und Aron Darmody als eine neue Art der Gouvernementalität verstehen. Ko-Kreation meint dabei „a sophisticated technology of government of consumers where the surplus value generated is based on appropriaton of the creative work of often networked and socially cooperative customers" (Zwick et al. 2008: 182). Mit dem Konzept der Gouvernementalität von Michel Foucault bezeichnen Zwick et al. in diesem Kontext die Hervorbringung von „particular forms of life in which costumers voluntarily provide unwaged and exploited, yet enjoyed labor" (Zwick et al. 2008: 176). Dies ist beispielsweise ebenjene Arbeit, die auch in den sozialen Netzwerken durch das Liken, Teilen und Kommentieren verrichtet wird. Hierbei handelt es sich nicht mehr um klassische disziplinarische Maßnahmen, die durch Androhung von Strafe und Verbot operieren, sondern um Erzeugung affektiver Gefüge, die Freiheiten dahingehend organisieren, dass sich bestimmte forms of life herausbilden. Doch operieren diese Prozesse der Ko-Kreation und der Erzeugung eines neuen Konsumsubjekts, welches gleichzeitig Produzent ist, nicht nur für digitale Güter. Ebenso wird für materielle Produkte, wie beispielsweise im Gestaltungsprozess von Spielzeug, diese Methodik angewandt. So bietet LEGO seinen User_innen die Möglichkeit, sich die Designsoftware herunterzuladen und eigene LEGO-Produkte zu konstruieren, die im nächsten Schritt zu einem hohen Preis bestellt werden können (Zwick et al. 2008: 181). Benutzer_innen wird dabei ein Bild von Freiheit und spielerischer Kreativität suggeriert. Dies stellt eine Organisation von Freiheit dar, die den „internen Regeln maximaler Ökonomie gehorcht“ (Foucault 2004: 436). Zwick et al. betonen dabei die oben diskutierte Vereinnahmung von Bereichen, die man klassischerweise außerhalb des Kapitalismus angesiedelt hätte: „[W]e see a co-creation economy as driven by the need of capital to set up processes that enable the liberation and capture of large repositories of technical, social, and cultural compe- tence in places previously considered outside of monetary value." (Zwick et al. 2008: 166) Es zeigt sich damit in der Ko-Kreation eine weitere Spielart der intensiven Expansion des Kapitalismus, der in der Verschiebung von Differenzen besteht, anstatt in der Erschließung einer noch vorhandenen Exteriorität. Daraus lässt sich schlussfolgern, dass sich die Idee der commons-basierten Peer-Produktion oder Ko-Kreation in diesem Sinne nicht mehr als wirksame Emanzipationsstrategie gegen den globalen Kapitalismus verstehen lässt. Die Dezentralisierung führt zwar zur in The Wealth of Networks angesprochenen Veränderung der Freiheit, jedoch ist diese neu gewonnene Freiheit, wie Zwick et al. betonen, nur eine von einem dezentralisierten Weltmarkt organisierte und modulierte Freiheit.

Einen etwas anders gelagerten Fall des Widerstandes, der sich vor allem auf das Ausnutzen von decodierten Strömungen der globalen Vernetzung bezieht, ist das Hackerkollektiv Anonymous. Der Fokus von Anonymous liegt vor allem darauf, sich für Redefreiheit und gegen Urheberrechte einzusetzen. Sie treten gegen global agierende Konzerne, staatliche Behörden und repressive Regime auf, wie beispielsweise die Unterstützung und Befürwortung des Arabischen Frühlings zeigt (Coleman 2015: 192). Entgegen den Ideen von Siefkes zeigt sich hierbei nicht die Annahme eines Außen des Kapitalismus, sondern ein Agieren innerhalb des kapitalistischen Gefüges. Die Abhängigkeit des globalen Kapitalismus auf digitale Vernetzung bringt decodierte Ströme in Form von Sicherheitslecks hervor, die von Anonymous ausgenutzt werden. Die Taktiken von Anonymous lassen sich hier mit Michel de Certeaus Alltagspraktiken des Widerstandes beschreiben, welche „Vorgehensweisen verfolgen, die der Disziplin entkommen, ohne jedoch ihren Einflußbereich zu verlassen“ (Certeau 1988: 197). Diese Strategien verfügen zwar über das Potenzial zur globalen Skalierbarkeit, jedoch scheint das Problem ihres Widerstandes im Sinne Certeaus letztlich darin zu bestehen, dass sie das Gefüge immer nur an seine Grenze, aber nie an die Schwelle bringen. Wie Certeau selbst schreibt, verlässt diese Widerstandsform nicht den Einflussbereich des Kapitalismus. Im Weiteren erzeugen die Ströme des Hacktivism neue Geldströme in den Sektor der Cyber Security und treiben damit weiter die weltweite Kriegsmaschine an, die diese Protestformen vereinnahmt.

Die beiden vorgestellten Beispiele, die einerseits ein Außen des Kapitalismus erzeugen zu versuchen und andererseits diesen von innen angreifen, haben natürlich ihre Legitimation als effektive temporäre 
oder lokale Widerstandsform, bleiben jedoch letztlich im Kapitalismus verankert und können seiner Vereinnahmung nicht entgehen. Beide Widerstandsstrategien bewegen sich in einem Rahmen, der mit der Stabilität der kapitalistischen Realität vereinbar bleibt. Diese Strategien setzen auf die Ausnutzung von Fluchtlinien, die sich jedoch durch die oben beschriebene Struktur des Kapitalismus immer vereinnahmen lassen. Ähnlich verhält es sich mit dem emanzipativen Subjekt von Hardt und Negri. Die Multitude stellt eine Fluchtlinie dar und kann somit immer von der kapitalistischen Vereinnahmung erfasst werden. Die virtuelle Multitude erschafft durch neue Subjektivierung eine lokale Exteriorität zum Kapitalismus (Hardt/Negri 2003: 402), die wie die oben diskutierten Beispiele wiederum deterritorialisiert und reterritorialisiert wird, anstatt sich als wirksamer Gegenspieler zum Kapitalismus zu etablieren. Eine anders strukturierte Emanzipationsform, die nicht über die Bahnung einer schöpferischen Fluchtlinie in ein post-kapitalistisches Gefüge operiert, präsentiert Tsing in The Mushroom at the End of the World.

\section{Von Pilzen und mechanischen Türken}

Die Besonderheit des von Tsing studierten MatsutakePilzes ist, dass dieser hauptsächlich in Gegenden auftritt, die durch menschliche Interventionen zerstört und ausgebeutet wurden. „Matsutake's willingness to emerge in blasted landscapes allows us to explore the ruin that has become our collective home." (Tsing 2015: 3) Paradoxerweise zählt der Matsutake gleichzeitig zu einem der teuersten Speisepilze der Welt. Daher hat sich in den Ruinen des Kapitalismus, wie Tsing sie nennt, eine eigene Dynamik im Zusammenspiel aus Pflücker_innen und Käufer_innen gebildet. "Matsutake resist the conditions of the plantation. They require the dynamic multispecies diversity of the forest - with its contaminating relationality." (Tsing 2015: 40) Dadurch widersetzt sich Matsutake der Skalierbarkeit, die für Tsing einen der Hauptgründe für den Erfolg der kapitalistischen Expansion darstellt. Mit Deleuze und Guattari könnte man sagen, Matsutake widersetzt sich der De- und Reterritorialisierung der kapitalistischen Kriegsmaschine, da er eben nicht in Territorien gedeihen kann, die einer permanenten Modulation unterliegen. Dahingehend ließe sich der Matsutake als eine Fluchtlinie verstehen. Doch denkt man an andere Luxusgüter, wie italienischen Parmesan, schottischen Whisky oder französischen Cham- pagner, so sind auch diese nur bedingt skalierbar und regional begrenzt. Die Besonderheit des Matsutakes liegt sicherlich darin, dass er in Regionen wächst, die vom Kapitalismus ausgebeutet und zurückgelassen wurden, jedoch kann er insofern eher als ein Bote aus einer zukünftigen post-kapitalistischen Welt angesehen werden. Der Matsutake könnte hier als Zeichen dafür gesehen werden, dass „die Erde [...] ihre eigenen Deterritorialisierungskräfte, ihre Fluchtlinien und ihre glatten Räume zur Geltung [bringt], die ihren Weg zu einer neuen Erde beleben und vorantreiben" (Deleuze/ Guattari 1992: 585).

\subsection{Prekarität und Veränderung}

Ein anderer Aspekt, den Tsing anspricht, ist jener der Prekarität. So schreibt sie: „What if precarity, indeterminacy, and what we imagine as trivial are the center of the systematicity we seek?" (Tsing 2015: 20) Prekarität ist für Tsing mit dem einschneidenden Ereignis der Atombombe in Hiroshima zu einer bestimmenden Konstante unserer Zeit geworden (Tsing 2015: 20). Anstatt jedoch deswegen Widerstände $\mathrm{zu}$ fordern, welche versuchen, dieser Prekarität zu entgehen, indem sie, wie in dem Beispiel von Siefkes, versuchen, ein Außen dieser prekären Gesellschaft zu errichten, pocht Tsing auf eine Affirmation dieser Prekarität. Die Matsutakepflücker_innen scheinen vollkommen im neoliberalen Kapitalismus gefangen zu sein und den Schwankungen des Marktpreises des Pilzes ausgeliefert. Nach Tsing befinden diese sich an der Schwelle in ein neues Gefüge. In genau diesen hyper-prekären Arbeitsverhältnissen, in denen die Pflücker_innen mit lebensbedrohlichen Konditionen konfrontiert sind, sieht Tsing Potenzial für Veränderung. Die Prekarität und Instabilität sind hierbei die bestimmenden Faktoren für einen Umbruch. „A precarious world is a world without teleology. Indeterminacy, the unplanned nature of time, is frightening, but thinking through precarity makes it evident that indeterminacy also makes life possible." (Tsing 2015: 20) In diesem Sinne ist es genau nicht der geplante Widerstand, sondern die Eröffnung neuer Möglichkeiten durch Erzeugung von Instabilität und die Betonung der Kontingenz der scheinbar stabilen und absoluten kapitalistischen Weltordnung. Um besser zu verstehen, inwiefern hier Prekarität bzw. ein Prekär-Werden im Rahmen von Deleuze und Guattari eine emanzipative Dimension besitzt und warum diese eine Instabilität erzeugt, die von den oben genannten Projekten nicht erreicht werden kann, ist es hilfreich, 
die Unterscheidung zwischen einer Destruktions- und einer Fluchtlinie einzuführen.

\subsection{Destruktions- statt Fluchtlinien}

Der Vorschlag von Tsing lässt sich mit einer Umarbeitung des Konzepts der Destruktionslinie beschreiben. Deleuze und Guattari bezeichnen als Destruktionslinie den Pol der Kriegsmaschine, der hervortritt, sobald die Kriegsmaschine vom Staatsapparat vereinnahmt wird und „der Krieg ihr Ziel“" wird (Deleuze/Guattari 1992: 584). Damit

„repräsentiert sie keineswegs das vermeintliche Wesen der Kriegsmaschine, sondern, wie mächtig sie auch sein mag, nur die Gesamtheit der Bedingungen, unter denen die Staaten sich diese Maschine auf die Gefahr hin aneignen, daß sie schließlich zum Horizont der Welt oder zur vorherrschenden Ordnung wird, in der die Staaten selber nur noch untergeordnete Teile sind." (Deleuze/Guattari 1992: 584)

Denkt man sich nun diese Anordnung der Kriegsmaschine des weltweiten Kapitalismus nicht durch die Staaten, sondern durch die Matsutakepflücker_innen, so repräsentieren diese hierbei die Extrembedingungen des globalen Kapitalismus. In dieser hyper-prekären Situation erzeugen sie jedoch eine Instabilität, die nicht zur „Bahnung einer schöpferischen Fluchtlinie“ (Deleuze/Guattari 1992: 584) durch die Kriegsmaschine führt, sondern zu einer Destruktionslinie, die eine unberechenbare Zukunft entwirft, in der diese Kriegsmaschine zerstört werden kann. Die Destruktionslinie unterscheidet sich von der Fluchtlinie also dadurch, dass letztere immer noch einer Vereinnahmung durch die bestehende Realität anheimfallen kann. Die Destruktionslinie hingegen ist eine (Zer-)Störung der kapitalistischen Realität, die durch genau diese Eigenschaft eine Instabilität erzeugt, die außerhalb einer jeden kapitalistischen Aneignung steht. Im Sinne dessen ließe sich Tsings Text als Appell für ein Prekär-Werdens verstehen. Das Paradoxe an den Matsutakepflücker_innen als Figuren der Schwelle ist jedoch, dass diese das unternehmerische Selbst par excellence darstellen und sich dennoch über die Grenze des Zugriffsbereichs der erneuten Vereinnahmung hinwegbewegen. Sie lassen dadurch die Kontingenz des kapitalistischen Gefüges offenbar werden. Damit versprechen sie keine konkrete Utopie, aber die konkrete Möglichkeit einer Endlichkeit der bestehenden Verhältnisse.

Während Hardt und Negri mit dem emanzipativen Subjekt der Multitude von einem noch eher klassischen
Ausbeutungsverhältnis ausgehen, lässt sich die Frage stellen, ob diese diffuse Reinkarnation der Dichotomie von Kapitalist_in und Proletarier_in sich noch wirklich anwenden lässt. Die Matsutakepflücker_innen entsprechen dabei doch eher den neoliberalen Arbeitsnomad_ innen, deren Tragödie es darstellt, nicht mehr in einer klassischen Arbeitssituation vom Kapital ausgebeutet zu werden (Mbembe 2017: 16), sondern sich in derartige prekäre und ungebundene Situationen begeben zu müssen, um sich gewissermaßen selbst auszubeuten. Die Pflücker_innen sind dadurch viel mehr auf beiden Seiten der Dichotomie zu verorten, womit sich die strengen Kategorien von Kapitalisten und Proletariat auflösen bzw. in ihrer Bedeutung für politische Instrumentalisierung abnehmen. Es zeigt sich also, dass dieser extreme Pol des globalen Kapitalismus mit Tsing affirmiert werden könnte, um Instabilitäten zu erzeugen. Sucht man nach derartigen prekären Arbeitssituationen, die gewissermaßen ihre eigene Destruktionslinie, im Sektor kognitiver Arbeit erzeugen, so bieten sich hier die Amazon Turker als Beispiel an.

\subsection{Abseits der Subjektivierung}

Im Jahr 2005 errichtet Amazon seinen digitalen Arbeitsmarkt Mechanical Turk. Auf diesem können Arbeiter_innen aus aller Welt rund um die Uhr sogenannte human intelligence tasks (HITs) ausführen, die von Firmen oder Einzelpersonen angeboten werden. Die Aufgaben der Arbeiter_innen, die auch Turker genannt werden, für diese HITs liegen in der Informations- und Bildersuche, dem Übersetzen von Texten, der Transkription von Audioaufnahmen, der Identifikation von Bildern oder der Beantwortung von Umfragen. Die Bezahlung für diese HITs schwankt zwischen einem Cent und einigen US-Dollar, je nach Zeitaufwand und Schwierigkeit der Aufgabe (Aytes 2003: 79-80). Hierbei ist zu beachten, dass diese Arbeitsverhältnisse der Turker kaum noch in ein gewöhnliches Schema von Anstellung hineinpassen. Wie Ross es treffend formuliert:

"The registered taskers of the Mechanical Turk and other e-lance operations would not be thought of as remotely resembling temporary employees any more than the uncompensated creatives who respond to the more skill-intensive kinds of crowdsourcing. They leave no trace of their employment, and certainly nothing to implicate an employer in any legal or regulated network of obligations. What they do, however, is bring the definition of a job much closer to its etymological source - a discrete lump, or 
piece, of work that exists only for the duration of its fulfillment." (Ross 2013: 20)

Die Arbeitsbedingungen der Amazon Turker sind hierbei unverkennbar nah an denen der Matsutakepflücker_innen, da auch sie keinerlei Garantie auf ihre Arbeit haben bzw. diese nur temporär existiert. Weiters ist zu beachten, dass die zu erfüllenden Aufgaben oftmals künstliche Intelligenzen (KI) trainieren, die in weiterer Folge diese Arbeiten übernehmen sollen. So liegt der Ursprung von Amazon Mechanical Turk darin, dass Amazon eine Plattform erstellen wollte, die eine Datenklassifizierung und -verarbeitung anbietet, die neueste KI noch nicht erfüllen konnte (Irani 2015: 225).

Aufgrund dieser Situation, dass sie eine KI trainieren, die ihre Arbeit übernehmen soll, schaffen sich die Turker durch ihren Arbeitsprozess gewissermaßen selbst ab. Sie tragen aktiv zur Automatisierung und Arbeitsplatzverminderung durch ihre Arbeit in hyperprekären Verhältnissen bei und erschaffen somit ihre eigene Destruktionslinie. Damit produzieren die Turker sich selbst als Instabilitäten im kapitalistischen Gefüge. Sie verschwinden als definierte Subjektivität innerhalb des gekerbten Raumes, der ihnen diese Subjektivität zuweist, in einen Bereich, in dem sie im gegenwärtigen Paradigma kaum noch integrierbar werden. Entgegen der Aufforderung zur „permanente[n] Weiterbildung“ (Deleuze 1993: 257), Anpassung und Transformation, also der ständigen neuen Subjektivierung, löschen sich die Turker selber aus. Sie begeben sich damit in einen Prozess des „Unwahrnehmbar-Werden“ (Deleuze/ Guattari 1992: 380), der sie im dynamischen Differenzraster zu einer Bedrohung für die bestehende Ordnung werden lässt, da sie die Kontingenz dieser Realität dadurch zur Erscheinung bringen. Im Unwahrnehmbar-Werden unterwandern die Turker die vorherrschenden Gefüge der Kontrolle, Organisation und Modulation. Genau diese Erzeugung von Instabilität durch eine Abschaffung der eigenen Arbeitsplätze und weitere Prekarisierung scheint mir der, von manchen Autoren_innen selbst eher vernachlässigte Appell des linken Akzelerationismus zu sein.

\section{Akzeleration und Automation}

In Inventing the Future formulieren Srnicek und Williams eine Kritik an den bisherigen Widerstands- und Emanzipationsideen im Kapitalismus, die sie des „folkpolitical thinking“ (Srnicek/Williams 2015: 9) beschuldigen. Sie sehen das Scheitern von linken Bewegungen in folgenden falschen Annahmen:
„In terms of spatial immediacy, folk politics privileges the local as the site of authenticity (as in the 100-miles diet or local currencies); habitually chooses the small over the large (as in the veneration of small-scale communities or local businesses); favours projects that are un-scalable beyond a small community (for instance, general assemblies and direct democracy); and often rejects the project of hegemony, valuing withdrawal or exit rather than building broad counter-hegemony." (Srnicek/Williams 2015: 11)

Die meisten der genannten Punkte treffen hierbei auch auf die oben beschriebenen Beispiele im kognitiven Kapitalismus zu. Als Gegenprojekt empfehlen Srnicek und Williams die Beschleunigung der Automatisierung von Arbeit und die Einführung eines Grundeinkommens.

Sie sprechen damit eine Idee an, die sich in dieser Form bereits bei Marx finden lässt: „Es bedarf Zeit und Erfahrung, bevor der Arbeiter die Maschinerie von ihrer kapitalistischen Anwendung unterscheiden und daher seine Angriffe vom materiellen Produktionsmittel selbst auf dessen gesellschaftliche Exploitationsform übertragen lernt." (Marx 1962: 452) Die geläufigere gegenwärtige Ausprägung einer solchen „Arbeiterempörung gegen die Maschinerie“ (Marx 1962: 452), die Marx hier anspricht, zeigt sich im Technikskeptizismus bzw. der Verurteilung der kapitalistischen Technologien als reine Kontrollinstrumente. Dass diese Anschuldigungen bis zu einem gewissen Grad äußerst zutreffend sind, zeigt sich an einigen der oben diskutierten Beispielen, wie der commonsbasierten Peer-Produktion oder der Hackerkultur. Deleuze und Guattari folgend besitzt jedoch ein jedes Gefüge immer die inhärente Tendenz zur Veränderung und seiner Auslöschung.

Srnicek und Williams selbst formulieren ihr Projekt entlang einer Hoffnung, die sich für sie durch die technologischen Errungenschaften der vergangenen Jahrzehnte aufgetan hat. Vor allem durch die immer besser werdende KI können viele der sogenannten kognitiven Arbeiten voraussichtlich bald von Maschinen übernommen werden. Ihre Forderungen formulieren Srnicek und Williams programmatisch wie folgt:

„With automation [...] machines can increasingly produce all necessary goods and services, while also releasing humanity form the effort of producing them. For this reason, we argue that the tendencies towards automation and the replacement of human labour should be enthusiastically accelerated and targeted as a political project of the left. This is a project that takes an existing capitalist tendency and seeks to push it beyond the acceptable 
parameter of capitalist social relations."(Srnicek/Williams 2015: 109)

Der letzte Satz des Zitates lässt bereits erkennen, dass es Srnicek und Williams hierbei nicht mehr um die Etablierung eines klassischen Antagonisten zum Kapitalismus geht, sondern eher um die Erzeugung einer Destruktionslinie, die das kapitalistische Gefüge jenseits seiner stabilen Parameter katapultiert. Eine solche Beschleunigung ist nicht eine, die einfach nur die Geschwindigkeit des Kapitalismus erhöht, sondern eine, „die auch navigiert, die ein experimenteller Entdeckungsprozess in einem universellen Möglichkeitsraum ist" (Srnicek/Williams 2013). Dies bedeutet, dass eine Akzeleration in diesem Sinne nicht einfach die Expansions- und Akkumulationstendenzen des Kapitalismus verstärkt, sondern gezielt die technologischen Bedingungen ausnutzt, um die Instabilitäten innerhalb des kapitalistischen Gefüges zu vermehren und somit eine offene Zukunft zu erzeugen. In diesem Sinne existiert jedoch nicht mehr ein virtuelles emanzipatives Subjekt, auf dessen Ankunft zu warten ist, sondern konkrete Subjekte in Extrempositionen der neoliberalen Gesellschaft.

Der Fehler, welchen die Autoren m. E. hierbei jedoch begehen, ist der, dass sie dem Übergangszustand in das neue vorgeschlagene Gefüge kaum Beachtung schenken bzw. außer Acht lassen, was es bedeutet, die Beschleunigung der Automation zu befürworten. Ihre Zukunftsvision besteht im Übertreten in einen neuen Zustand, der sich selbst wiederum absolut und stabil setzt, der sich jedoch dann nicht mehr in der Form eines globalen Kapitalismus, sondern eines Luxuskommunismus präsentiert. Die konkrete Utopie steht für die Autoren mehr im Vordergrund als die Prekarisierung, die eine unweigerliche Folge der Selbstabschaffung von Arbeitsplätzen durch einen derartigen Gebrauch der technologischen Bedingungen ist. Die Amazon Turker erzeugen in ihrer Arbeit nicht eine konkrete Utopie, sondern brechen nur mit der scheinbaren Stabilität der kapitalistischen Realität. Der Weg, der deswegen eher zu besprechen wäre als die Vorwegnahme einer ungewissen Zukunft durch eine konkrete Utopie, wäre jener, den Tsing vorschlägt, nämlich die Erschaffung einer Welt ohne Teleologie, die eben genau in Prekarität als Konstante unserer Zeit ersichtlich wird. Die Frage, die es schlussendlich noch genauer zu diskutieren gilt, ist, worin nun Gefahren und Potenziale dieser Akzeleration der Prekarität liegen.

\section{Emanzipation durch Hyper-Prekarisierung?}

Eine Kritik, die man an Srnicek und Williams richten sollte, ist, dass sie einerseits eine implizite Verelendung mit gravierenden Folgen vorschlagen und andererseits quasi-messianische Hoffnungen auf eine technologische Utopie erzeugen. Letzteres Problem tritt ebenfalls bei Hardt und Negri auf, die zwar davon sprechen, dass es sicherlich einen Augenblick geben muss, an dem die Wiederaneignung durch die Multitude erfolgt (Hardt/ Negri 2003: 417), aber die quasi-messianische Hoffnung auf dessen Ankunft ebenso voraussetzen wie den Luxuskommunismus von Srnicek und Williams. Das Potenzial ihres spekulativen Projekts besteht genau darin, diese Erzeugung von Instabilitäten innerhalb einer kapitalistischen Realität, die ich oben in gewissen hyper-prekären Arbeitsverhältnissen schon vereinzelt aufzeigt habe, zu beschleunigen und damit keine neue Teleologie einzuführen. Anstatt also die technologische Utopie des Akzelerationismus zu affirmieren, sollte der Fokus eher darauf liegen, das kapitalistische Gefüge zum Implodieren zu bringen, indem man Destruktionslinien erzeugt, die nicht mehr einfach vereinnahmt werden können, sondern die Spannungsverhältnisse und unerwünschte Differenzen in den globalen Kapitalismus einführen. Der Fokus eines solchen emanzipativen Projekts würde darauf zielen, den von Armen Avanessian so bezeichneten spekulativen Zeitkomplex zu unterwandern.

\subsection{Wider den spekulativen Zeitkomplex}

Trotz seiner enormen Geschwindigkeit und des futuristischen Innovationsdenkens erschafft der globale Kapitalismus alles andere als einen offenen Zukunftshorizont. Im Gegenteil zeichnen sich zeitgenössische Entwicklungen eher dadurch aus, dass ein spekulativer Zeitkomplex durch eine Vereinnahmung der Zukunft konstruiert wird. Der spekulative Zeitkomplex bezeichnet den Verlust der „Gegenwart als primäre Kategorie der menschlichen Erfahrung [und] als Grundlage sowohl für das Verständnis der Zeit, als auch dessen, was Zeit ist“ (Avanessian/Suhail 2016: 7-8). Dieser manifestiert sich in der präemptiven Operationslogik des Kapitalismus dahingehend, dass die Zukunft sich vor der Gegenwart ereignet (Avanessian/Suhail 2016: 7). Ein alltägliches Beispiel hierfür ist das im zweiten Abschnitt bereits genannte, dass Amazon nicht nur Artikel vorschlägt, die zu den eigenen Interessen passen, sondern ebenfalls solche, die auf zukünftige 
Präferenzen antworten, ihnen bestenfalls zuvorkommen. Damit kommt es zu einer „Vorwegnahme der Zukunft“ (Avanessian/Suhail 2016: 10) unter dem Ausschluss alternativer Zukünfte. Ähnlich verhält es sich mit der oben diskutierten Gouvernementalität der Ko-Kreation, in welcher eine bestimmte Lebensform in der Gegenwart, aber auch der Zukunft durch gezielte Modulation und Kontrolle von Subjektivierungsprozessen vorweggenommen wird.

Eine Beschleunigung der Prekarität bzw. ein akzeleriertes Prekär-Werden würde nicht darauf abzielen, Fluchtlinien zu produzieren, die genauso schnell wieder vereinnahmt werden und damit erfolglos bleiben, sondern alternative und offene Zukünfte zu erzeugen. Der Plural von Zukunft verweist hierbei explizit darauf, dass die einheitliche, globale Zukunft nur ein Produkt der weltumspannenden Kriegsmaschine darstellt. Damit wäre ein solches Projekt zwar gegenhegemonial in dem Sinne, dass es die bestehende hegemonielle Weltordnung zerstört, aber damit einher geht nicht die Etablierung einer neuen hegemonialen Ordnung, sondern die Öffnung für eine kommende Pluralität. Warum Prekarität als solche hierfür ein geeignetes Mittel darstellt, um die kapitalistische Realität zu destabilisieren, lässt sich an Judith Butlers Arbeiten zur Wirkmächtigkeit von Prekarität näher erläutern.

\subsection{Störung der hegemonialen Realität}

Für Butler stellt Prekarität nicht einfach nur eine existenzielle Kondition von Individuen dar, sondern eine soziale Kondition, von welcher eine klare politische Forderung ausgeht (Butler 2016: xxv). Mit Hilfe von Emmanuel Levinas' phänomenologischen Überlegungen zum Anderen entwickelt Butler die Position, dass wir im Antwortenmüssen auf das Antlitz des Anderen die Prekarität des Anderen, eines anderen Lebens, erkennen und dies einen Handlungsappell darstellt (Butler 2006: 134). Dies beschreibt sie folgendermaßen: "The face of the Other comes to me from outside, and interrupts the narcissistic circuit. The face of the Other calls me out of narcissim towards something finally more important." (Butler 2006: 138) Der Andere, der mir als prekär erscheint, bricht damit mit dem neoliberalen Individualismus. Dieser Andere verweist auf das Bestehen einer Gemeinschaft, auf das Soziale (Butler 2016: 14) und etwas vor jeder Subjektivierung, Identität und jedem Multikulturalismus Liegendes (Butler 2016: 32).
Ähnlich gelagert ist dies in Deleuzes letztem zu Lebzeiten verfassten Text „Die Immanenz: ein Leben“. So verweist er hier ebenfalls auf die Auflösung der Subjektivität und das Erscheinen eines präsubjektiven Lebens in einer Situation der Prekarität und Verwundbarkeit. Auf Charles Dickens' Unser gemeinsamer Freund referierend, schreibt er: „Ein Schurke, ein übles, von allen verachtetes Subjekt wird sterbend hereingebracht und mit einem Mal bezeugen alle, die ihn pflegen, eine Art Eifer, Achtung, Liebe gegenüber dem geringsten Lebenszeichen des Sterbenden." (Deleuze 2005: 367) Das Antlitz des Sterbenden verweist auf die eigene Prekarität, lässt die üblichen Kerbungen des gesellschaftlichen Feldes kurzzeitig brüchig werden und fordert zur Sorge und Handlung auf. Ebenso verhält es sich mit Bildern von brennenden und sterbenden Kindern im Vietnamkrieg. Diese entfalteten, so Butler, folgende Wirkungskraft: „The images furnished a reality, but they also showed a reality that disrupted the hegemonic field of representation itself." (Butler 2006: 150) Die Differenzen in der Ontologie von Deleuze und Butler ignorierend lässt sich also feststellen, dass Prekarität zu einer Störung der hegemonialen Realität führt.

Geht man nun also von einer Vermehrung von Prekarität durch Beschleunigung der Verelendung und Prekarisierung aus, so stellt diese dadurch entstehende Prekarität eine politische Forderung. „The precarity of life imposes an obligation upon us" (Butler 2016: 2), schreibt Butler. Diese Forderung ist eine, die zum Handeln aufruft und gleichzeitig die Kontingenz der bestehenden Realität hervorhebt. Prekarität eröffnet in diesem Sinne einen Bruch mit dem spekulativen Zeitkomplex und bringt die Vereinnahmungsmechanismen des Kapitalismus zum Stocken.

Akzeleration zur Erzeugung einer Destruktionslinie, in diesem Falle jene Destruktionslinie der Prekarität, scheint aus dieser Perspektive ein Weg zu sein, um die Möglichkeit einer wie auch immer gearteten post-kapitalistischen Gesellschaft zu eröffnen. Prekarität stellt für die erneute Eröffnung einer offenen und kontingenten Zukunft ohne einen konstruierten Telos vermutlich nicht die einzig mögliche Emanzipationsstrategie dar, jedoch müssten sich ähnliche Ansätze ebenfalls von der Idee einer konkreten Utopie, die wiederum einen neuen metastabilen Zustand als absoluten setzt, verabschieden. Nichtsdestotrotz ist hierbei die extreme Ungewissheit dieser Widerstandsstrategie $\mathrm{zu}$ beachten. Sie fordert aktiv auf, alles zu riskieren und eine maximal ungewisse Zukunft zu entwerfen, um den spekulativen Zeitkomplex des Kapitalismus zu destabi- 
lisieren. Das Ende des Kapitalismus kann demzufolge durch eine beschleunigte Hyper-Prekarisierung passieren, welche vollkommen neue Gefüge hervorbringen kann, indem sie das Bestehende durch eine Beschleunigung seiner Destruktionslinie zerstört. Niemand kann jedoch wissen, was eine solche Zukunft bringen mag. Eine Wiederaneignung der Zukunft ermöglicht jedoch, sich wieder das Ende des Kapitalismus vorzustellen.

\section{Literatur}

Adkins, B. (2015): Deleuze and Guattari's A Thousand Plateaus. A Critical Introduction and Guide. Edinburgh: Edinburgh University Press.

Avanessian, A./Malik, S. (2016): Der Zeitkomplex. In: dies. (Hg.): Der Zeitkomplex. Postcontemporary. Berlin: Merve, 7-36.

Aytes, A. (2013): Return of the Crowds. Mechanical Turk and Neoliberal States of Exception. In: Scholz, T. (Hg.): Digital labor: the Internet as playground and factory. New York: Routledge, 79-97.

Benkler, Y. (2006): The Wealth of Networks. How Social Production Transforms Markets and Freedom. New Haven/ London: Yale University Press.

Butler, J. (2006): Precarious Life. The Powers of Mourning and Violence. London/New York: Verso.

Butler, J. (2016): Frames of War. When Is Life Grievable. London/New York: Verso.

Certeau, M. d. (1988): Kunst des Handelns. Berlin: Merve.

Coleman, G. (2015): Hacker, Hoaxer, Whistleblower, Spy. The Many Faces of Anonymous. London/New York: Verso.

Corsani, A. (2012): Gibt es einen kognitiven Kapitalismus? Elemente eines Bruchs. In: Lorey, I./Neundlinger, K. (Hg.): Kognitiver Kapitalismus. Es kommt darauf an 13. Wien/Berlin: Turia + Kant, 103-120.

Deleuze, G./Guattari, F. (1992): Tausend Plateaus. Berlin: Merve.

Deleuze, G. (1993): Postskriptum über die Kontrollgesellschaften. In: ders.: Unterhandlungen 1972-199o. Frankfurt/M.: Suhrkamp, 254-262.

Deleuze, G. (2005): Die Immanenz: ein Leben. In: ders.: Schizophrenie und Gesellschaft. Texte und Gespräche 19751995. Frankfurt/M.: Suhrkamp, 365-370.

Fisher, M. (2009): Capitalist Realism. Is There Not Alternative? Winchester/Washington: Zero Books.

Foucault, M. (2004): Die Geschichte der Gouvernementalität II. Die Geburt der Biopolitik. Vorlesungen am Collège de France 1978-1979. Frankfurt: Suhrkamp.

Hardt, M./Negri, A. (2003): Empire. Die neue Weltordnung. Frankfurt/New York: Campus.
Irani, L. (2015): Difference and Dependence among Digital Workers: The Case of Amazon Mechanical Turk. The South Atlantic Quarterly, 114 (1), 225-234.

Lazzarato, M. (1998): Immaterielle Arbeit: Gesellschaftliche Tätigkeit unter den Bedingungen des Postfordismus. In: Atzert, T. (Hg.): Umherschweifende Produzenten. Immaterielle Arbeit und Subversion. Berlin: ID, 39-52.

Marx, K. (1962): Das Kapital. Kritik der politischen Ökonomie. Buch I: Der Produktionsprozess des Kapitals. Berlin: Dietz.

Mbembe, A. (2017): Kritik der schwarzen Vernunft. Berlin: Suhrkamp.

Ross, A. (2013): In Search of the Lost Paycheck. In: Scholz, T. (Hg.): Digital labor: The Internet as playground and factory. New York: Routeledge, 13-32.

Siefkes, C. (2016): Eine Welt, in der alle gut leben können. Das Potential der commonsbasierten Peer-Produktion. In: Baier, A./Hansig, T./Müller, C./Werner, K. (Hg.): Die Welt reparieren: Open Source und Selbermachen als postkapitalistische Praxis. Bielefeld: Transcript, 63-70.

Srnicek, N./Williams, A. (2013): \#Beschleunigungsmanifest für eine akzelerationistische Politik. Online: https:// syntheticedifice.files.wordpress.com/2013/o6/beschleunigungsmanifest.pdf [12.2.2019].

Srnicek, N./Williams, A. (2015): Inventing the Future. Postcapitalism and a World without Work. London/New York: Verso.

Tsing, A. L. (2015): The Mushroom at the End of the World. On the Possibility of Life in Capitalist Ruins. Princeton/ Oxford: Princeton University Press.

Vogl, J. (2010): Das Gespenst des Kapitals. Zürich: Diaphanes.

Young, E. B./Genosko, G./Watson, J. (2013): The Deleuze \& Guattari Dictionary. London/New York: Bloomsbury.

Zwick, D./Bonsu, S.K./Darmody, A. (2008): Putting Consumer to Work. ,Co-creation' and new marketing governmentality. Journal of Consumer Culture, 8 (2), 163-196. 